\title{
Exploring Phonon Signals by High Energy / High Spatial Resolution EELS
}

\author{
O.L. Krivanek ${ }^{1,5}$, N. Dellby ${ }^{1}$, T.C. Lovejoy ${ }^{1}$, N.J. Bacon ${ }^{1}$, G.J. Corbin ${ }^{1}$, P. Hrncirik ${ }^{1}$, Z.S. Szilagyi ${ }^{1}$, T. \\ Aoki $^{2}$, R.W. Carpenter ${ }^{2,3}$, P.A. Crozier ${ }^{2,4}$, J. Zhu ${ }^{2,4}$, P. Rez ${ }^{2,5}$, R.F. Egerton ${ }^{6}$, and P.E. Batson ${ }^{7}$ \\ ${ }^{1}$ Nion Co., 1102 Eight St, Kirkland, WA 98033, USA \\ ${ }^{2}$ Center for Solid State Science, Arizona State University, Tempe, AZ 85287, USA \\ ${ }^{3}$ Department of Chemistry and Biochemistry, Arizona State University, Tempe, AZ 85287, USA \\ ${ }^{4}$ School for Engineering of Matter, Transport and Energy, ASU, Tempe, AZ 85287, USA \\ ${ }^{5}$ Department of Physics, ASU, Tempe, AZ 85287, USA \\ ${ }^{6}$ Department of Physics, University of Alberta, Edmonton T6G 2E1, Canada \\ ${ }^{7}$ IAMDN, Department of Physics, Rutgers University, Piscataway, NJ 08854, USA
}

Using the High Energy Resolution Monochromated EELS-STEM (HERMES) instrument we have developed [1] together with a Gatan Enfinium spectrometer retrofitted with extra-stable power supplies [2], we now achieve sub-20 meV resolution in electron energy loss spectra (EELS) acquired in $>1 \mathrm{~s}$. We have also been able to improve the rate of the decay of the zero loss peak (ZLP) such that tail intensities of $<4 \times 10-4$ of the ZLP maximum have been recorded at energy losses as small as $100 \mathrm{meV}$.

Besides the high energy resolution and minimized tail ZLP, HERMES provides several new capabilities, including: 1) spatial resolution - we can record full-resolution spectra from sample areas as small as a few Á; 2) flexibility - we can also record spectra from areas as large as $10 \mu \mathrm{m}$, and attain an angular resolution of $\sim 1 \mu \mathrm{rad}$ (using a camera length setting of $\sim 1 \mathrm{~km}$ ); 3) angular acceptance - we can attain a good energy resolution with EELS acceptance angles of $\pm 30 \mathrm{mrad}$ and greater; and 4) brightness - we use a bright cold field emission (CFE) gun, and can therefore extract appreciable signals from Å-sized areas. Taken together, these capabilities promise that signals not accessible in electron microscopy before are about to become available. Here we explore phonon spectroscopy; in a related contribution at this meeting, we explore detecting $\mathrm{H}$ and other light elements by energy-filtered HADF imaging [3].

Fig. 1 illustrates how important signals have up to now been "hidden in plain sight" - obscured by a broad zero loss peak. The solid green spectrum was recorded in $0.13 \mathrm{~s}$ at $60 \mathrm{keV}$, with no sample, and with the beam passing through the monochromator but the energy-selecting slit retracted. The ZLP FWHM is $\sim 250 \mathrm{meV}$, typical of tungsten CFE operated with a total emission current $<1 \mu \mathrm{A}$. The red (line) spectrum was recorded with the slit in, in $0.1 \mathrm{~s}$, and shows FWHM of $14 \mathrm{meV}$. It demonstrates the $\sim 20 \mathrm{x}$ improvement in energy resolution that's readily available with our system.

The blue spectrum in Fig. 1 was recorded from a $\sim 2 \mathrm{~nm} \varnothing$ area in $\mathrm{SiO}_{2}$, in a single acquisition of $10 \mathrm{~s}$ duration, with a beam current of $\sim 10 \mathrm{pA}$, probe convergence angle of $\pm 12 \mathrm{mrad}$ and collection angle also $\pm 12 \mathrm{mrad}$. Its ZLP (not shown) had a FWHM of $\sim 17 \mathrm{meV}$. The optical phonon peak visible at 140 meV energy loss had a peak intensity of $\sim 1 \times 10^{-4}$ (above background) relative to the zero loss peak, a level the tail of the unmonochromated ZLP only decays to at about $1.7 \mathrm{eV}$. The energy of the phonon peak is in good agreement with the energy of the strongest feature normally observed in infrared spectra of $\mathrm{SiO} 2$, at $1100 \mathrm{~cm}^{-1}$. (To convert $\mathrm{cm}^{-1}$ to $\mathrm{meV}$, divide by 8 .)

The phonon signal is due to two types of interactions: a short-range and a long-range one. Fig. 2 demonstrates that both types of signals can appear in a single line scan. Fig. 2(a) shows a high-angle 
annular dark field (HAADF) image of the examined area in a $\mathrm{Si}_{-} \mathrm{SiO}_{2}$ sample, with the scan line used for collecting EELS data marked. The scan started in the $\mathrm{Si}$, went through the $\mathrm{SiO}_{2}$ layer, and ended in the vacuum outside the sample. Fig. 2(b) shows the integrated intensity of the background-subtracted $\mathrm{SiO}_{2}$ phonon peak (as a fraction of the ZLP intensity) in a series of 100 spectra recorded in $10 \mathrm{~s}$ each, taken as the beam progressed along the line. The figure also shows the sample thickness, determined by taking the ratio of the intensity of energy losses, recorded separately up to $180 \mathrm{eV}$, to the ZLP, and assuming a mean free path of $72 \mathrm{~nm}$ for $\mathrm{SiO}_{2}$. Two sample areas are especially interesting: the $\mathrm{Si}-\mathrm{SiO}_{2}$ interface, where the phonon intensity goes to zero $\sim 3 \mathrm{~nm}$ inside the $\mathrm{Si}$ and builds up only gradually inside the $\mathrm{SiO}_{2}$, and the $\mathrm{SiO}_{2}$-vacuum interface, which shows an initial sharp drop-off and then a long decay tail stretching tens of nanometers outside the sample. The long tail suggests that phonon spectroscopy can be carried out in the STEM with an aloof beam, thus avoiding damage in radiation-sensitive samples.

We have also recorded optical phonons in several other materials, measured the phonon energy varying with the scattering angle and the probe position, and observed an enhancement of the short-range signal at high scattering angles. Results illustrating these phenomena will be shown at the meeting.

\section{References:}

[1] OL Krivanek et al., Microscopy 62 (2013) 3-21.

[2] OL Krivanek et al., Proceedings 2013 EMAG meeting, in print (2014).

[3] TC Lovejoy et al., these proceedings (2014).

[4] We gratefully acknowledge the use of facilities within the LeRoy Eyring Center for Solid State Science at ASU, and grants NSF MRI-R2 \#0959905, DE-SC0004954 and DE-SC0007694.

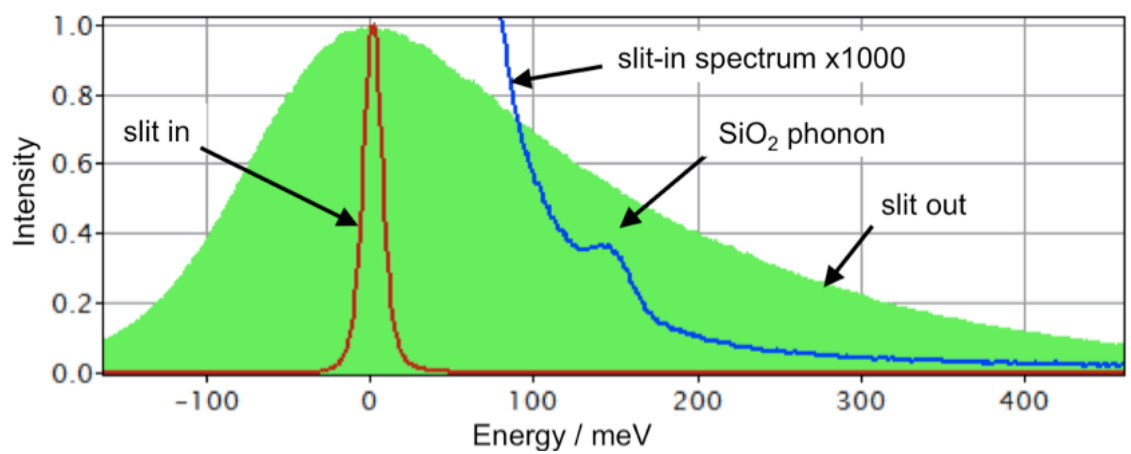

Figure 1. EEL spectra recorded with the monochromator slit out (green) and in (red and blue).
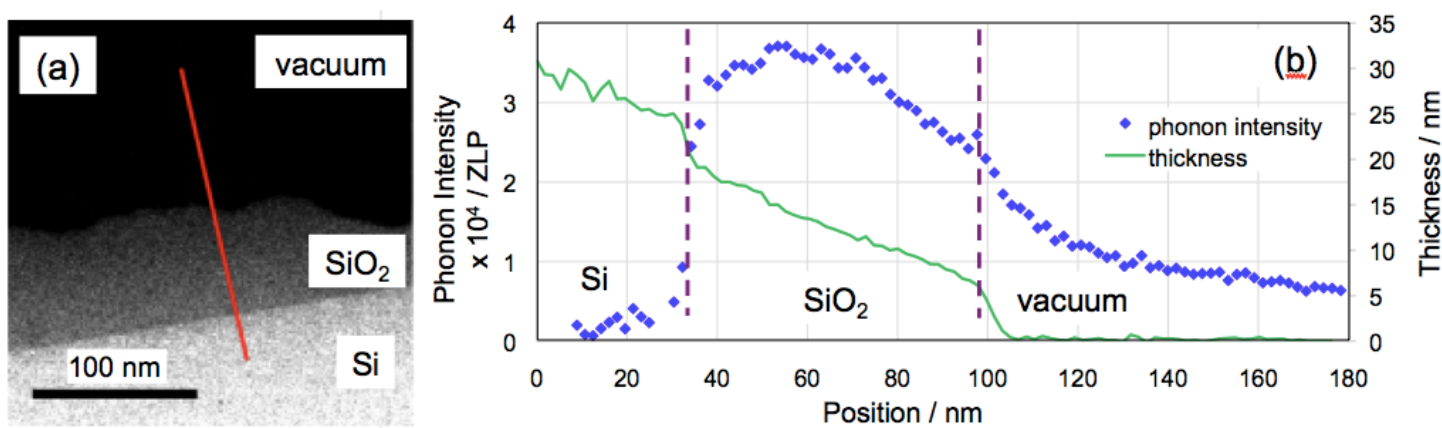

Figure 2. Variation of the phonon signal intensity in a line profile taken from $\mathrm{Si}$ via $\mathrm{SiO}_{2}$ into vacuum. $60 \mathrm{keV}, 10 \mathrm{pA}$ probe current. (a) HAADF image showing profile location. (b) Profile showing the phonon intensity and the sample thickness. Sample courtesy Dr. John Bruley, IBM. 